\title{
Measurement of Uniformity and Multiple Head Registration (MHR)/Centre of Rotation (COR) of a Newly Installed Hybrid SPECT/CT
}

\section{At INMAS, Mymensingh}

\author{
${ }^{1}$ Md. Fakhar Uddin, ${ }^{1}$ S M Moinul Islam, ${ }^{1} \mathrm{M}$ Nasim Khan, ${ }^{1}$ Ratan Kumar Chakraborty, ${ }^{1}$ ShakilaZaman \\ Rima, ${ }^{1}$ Rawshan Ara, ${ }^{1}$ Nazmun Nahar, ${ }^{2}$ Rajada Khatun \\ ${ }^{1}$ Institute of Nuclear Medicine \& Allied Sciences, Mymensingh \\ ${ }^{2}$ Accelerator Facilities Division, AEC, Dhaka
}

Address for Correspondence: Md. Fakhar Uddin, Scientific Officer, Institute of Nuclear Medicine \& Allied Sciences, Mymensingh, Cell phone: +880 1813677091, E-mail: sazid_cu07@yahoo.com

\begin{abstract}
The main quality controls of hybrid SPECT/CT are Uniformity and Multiple Head Registration (MHR)/Centre of rotation (COR). Uniformity corrects and verifies camera's response to uniform distribution of radionuclides on the detector surface. MHR/COR not only observes mechanical errors but also quantitatively corrects the errors due to movement of patients and detectors onthe SPECT images. The aim of the study is to establish baseline values thatcan be used as reference for future quality assurance and to verify the manufacturer's specification. Both intrinsic and extrinsic Uniformity were measured by using $18 \mu \mathrm{Ci}{ }^{99 \mathrm{~m}} \mathrm{Tc}$ point source and $35 \mathrm{mCi}{ }^{99 \mathrm{~m}} \mathrm{Tc}$ flood source respectively. For MHR/COR measurements, MHR Phantom containing five approximately equal strength ( $1 \mathrm{mCi}^{99 \mathrm{~m}} \mathrm{Tc}$ of each) point sourceswere used. The values were found to be in correlation with manufacturer's specification.
\end{abstract}

Key Words: SPECT/CT, QC, Uniformity, MHR/COR and Calibration

\section{INTRODUCTION}

There are three Hybrid SPECT/CTcurrently in operation in Bangladesh. The SPECT/CT installed in Mymensingh has the technology to deliver exact anatomic localization of region of interest. This work was pioneered by Lang and Hasagawa et al (1). However, if quality control (QC)to detect change in the performance of a gamma camera system is not done properly, it will adversely affect the interpretation of clinical studies. There are several QC procedures prescribed 
by different organization such as National Electrical Manufacturers Association (NEMA), the American Association of Physicists in Medicine (AAPM), and other regulatory, advisory, and professional organizations like IAEA guidelines. In Bangladesh, IAEA guidelines are followed for QC of most nuclear medicine equipment. After installation, a series of quantitative and qualitative performance tests known as acceptance tests are performed for quality assurance. Among them, Uniformity, both intrinsic and extrinsic, and Multiple Head Registration (MHR)/Centre of rotation (COR) were performed. Uniformity is most commonly practiced among nuclear medicine QC procedures. It is a measure of camera's response to uniform distribution of radioactivity on the detector surface. Perfectly uniform image is the consequence of its ideal response (2). This establishes a baseline for uniformity correction, and confirms that the detector is free of major defects. The extrinsic flood calibration verifies that the collimators can produce uniform floods and have not been damaged during shipment.

\section{Basic Concept}

Uniformities are quantified by integral uniformity (IU) and differential uniformity (DU) for both useful field of view (UFOV) and central field of view (CFOV). UFOV is the detector area that is used for imaging, and CFOV is the $75 \%$ of the useful FOV. In each of these fields of views IU is calculated by using Equation 1(3).

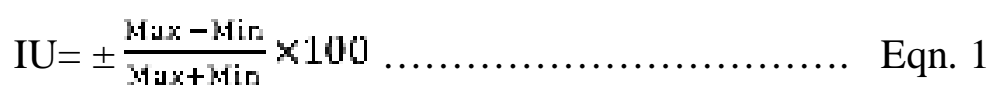

Where Max is the maximum counts and Minis the minimum counts in the pixels lying within the UFOV and the CFOV.

DU is expressed as the following Equation 2(4).

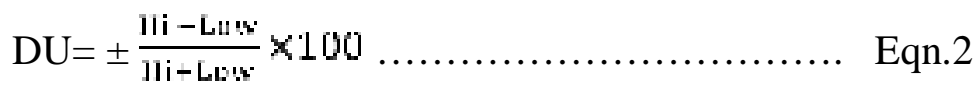

Here, $\mathrm{Hi}$ is the maximum \% pixel counts, and Low is the minimum \% pixel counts, for all rows and columns within a localized line of pixels; (Hi-Low) is the largest deviation.

Multiple Head Registration / Center of Rotation (MHR/COR) is another important QC for SPECT/CT system. Normally, COR is used for non-integrated systems and MHR is used for integrated systems. It observes mechanical errors in gamma camera as well as corrects, quantitatively, the errors from the movement of patients and detectors on the SPECT image (5). MHR/COR includes center of rotation, axial shift and back projection angle etc. COR corresponds to offset of detector centre relative to system centre of rotation, whereas axial shift 
indicates axial offset of detectors relative to each other and back projection angle is angular offset of detectors relative to each other (6). If, however, deviation arises beyond the permissible limit either in uniformity or in MHR/COR it needs correction.

The aim of this study was to establish baseline values of uniformity and MHR/COR that will be used as reference for future quality control and also verify manufacturer's specification.

\section{MATERIALS AND METHODS}

\section{Uniformity}

Uniformity test was performed intrinsically and extrinsically for our dual head Symbia T-series SPECT/CT.

Intrinsic uniformity: Intrinsic uniformity is done without collimator to monitor the condition of sodium iodide crystal and internal electronics. It is measured in terms of integral uniformity (IU) and differential uniformity (DU). The following procedures were followed to measure intrinsic uniformity.

Methods for Intrinsic Uniformity were performed according to the Manufacturer's procedures and guidelines.

\section{Verification:}

For intrinsic verification, second static acquisition was taken for 50,000 kcts in both detectors simultaneously and then corrected flood was calculated.

Extrinsic Uniformity: A rectangular shape fillable flood phantom wasused that contained uniformly distributed $35 \mathrm{mCi}{ }^{99 \mathrm{~m}} \mathrm{Tc}$ mixed with water uniformly to verify the detectors performance. Extrinsic uniformity was performed according to the Manufacturer's procedures and guidelines. A collimator was used in this study.

Verification: For extrinsic verification, two static acquisitions of 10 million counts of each were taken separately for both detectors and flood calculation activity was performed. Other set up were same as mentioned earlier.

\section{Multiple Head Registration (MHR)/Centre of Rotation (COR) Calibration:}

MHR/Recalibration was performed for LEAP collimator and this configuration is used clinically. This calibration aligns the detector to the gantry's centre of rotation and to one another.

Methods for MHR/COR was performed according to the Manufacturer's procedures and guidelines. 


\section{RESULTS}

Uniformity: Uniformities were found quantitatively in terms of integral and differential uniformity. The values are shown in Table 1.

Table1: Quantitative values of intrinsic and extrinsic uniformity

\begin{tabular}{lccccc}
\hline \multirow{2}{*}{ Detector } & Manner of & \multicolumn{2}{c}{ Intrinsic Uniformity } & & \multicolumn{2}{c}{ Extrinsic Uniformity } \\
\cline { 5 - 6 } & Uniformity & CFOV & UFOV & CFOV & UFOV \\
\hline Detector 1 & Integral & $1.33 \%$ & $1.99 \%$ & $2.95 \%$ & $3.15 \%$ \\
& & & & $2.14 \%$ & $2.14 \%$ \\
Detector 2 & Differential & $1.00 \%$ & $1.41 \%$ & $2.30 \%$ & $2.69 \%$ \\
& Integral & $1.41 \%$ & $1.45 \%$ & $1.93 \%$ & $2.12 \%$ \\
\hline
\end{tabular}

\section{Multiple Head Registration (MHR)/Centre of Rotation (COR):}

MHR/COR calibration were done at 3 distinct configuration angles $180^{\circ}, 90^{\circ}$ and $76^{\circ}$ for LEAP as shown in Table 2.

Table 2: MHR/COR performance values

\begin{tabular}{lcccccc}
\hline $\begin{array}{l}\text { Detector's } \\
\text { Orientation } \\
\text { Detector Name }\end{array}$ & \multicolumn{2}{c}{ At $180^{\circ}$} & \multicolumn{2}{c}{ At $90^{\circ}$} & \multicolumn{2}{c}{ At $76^{\circ}$} \\
\hline $\begin{array}{l}\text { Centre of } \\
\text { Rotation (in mm) }\end{array}$ & 0.412 & -1.136 & 1.060 & 0.093 & 0.468 & -0.819 \\
$\begin{array}{l}\text { Axial shift } \\
\text { (in mm) }\end{array}$ & 0.268 & -0.268 & -0.326 & 0.326 & 0.212 & -0.212 \\
$\begin{array}{l}\text { Back Projection } \\
\text { Angle (in degree) }\end{array}$ & 0.014 & -0.014 & 0.227 & -0.227 & 0.039 & -0.099 \\
$\begin{array}{l}\text { System } \\
\text { Resolution at 20 } \\
\text { cm (in mm) }\end{array}$ & 18.968 & 18.921 & 20.635 & 20.672 & 20.596 & 20.584 \\
\hline
\end{tabular}

Plus/minus indicates the left and right side. 
Flood images showed excellent visual acceptance (Figure $1 \& 2$ ).

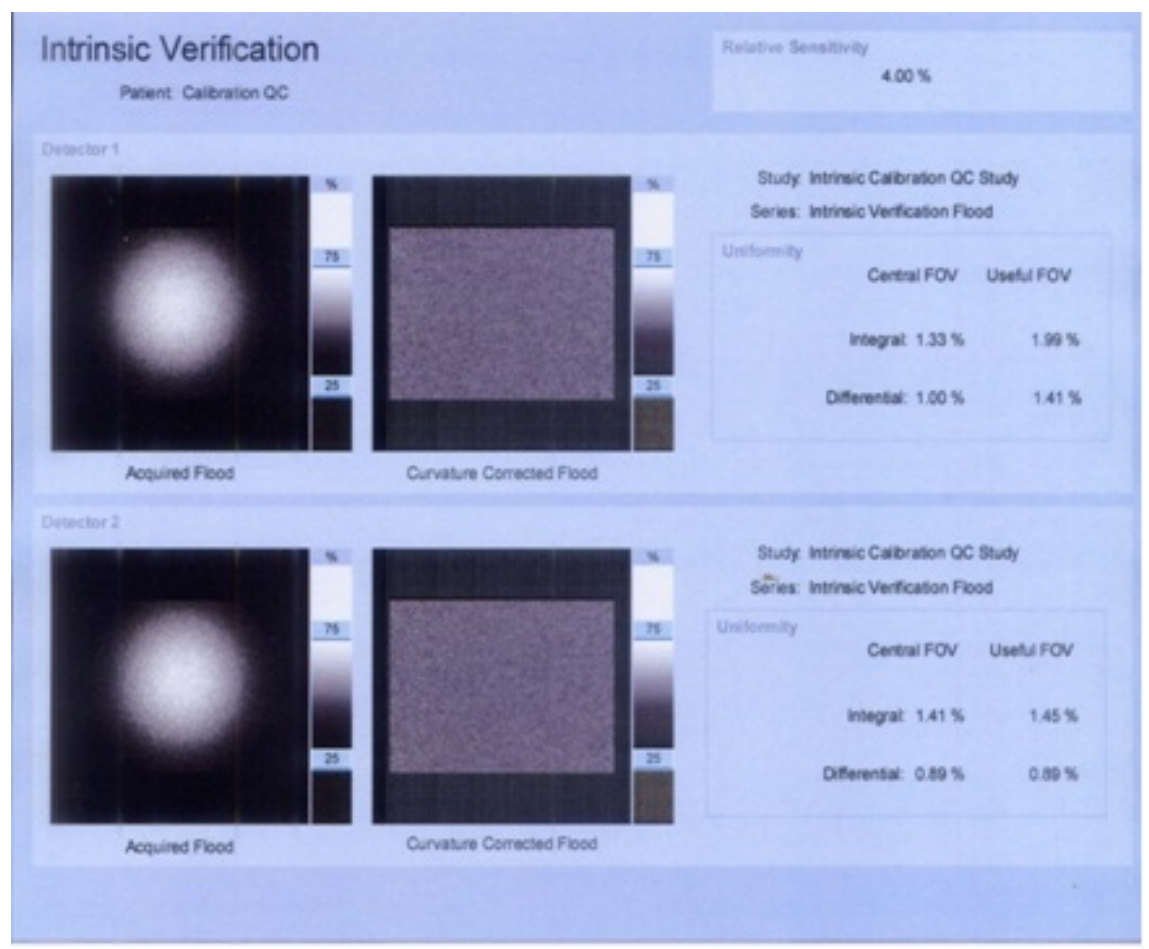

Figure 2: Extrinsic flood calculation image

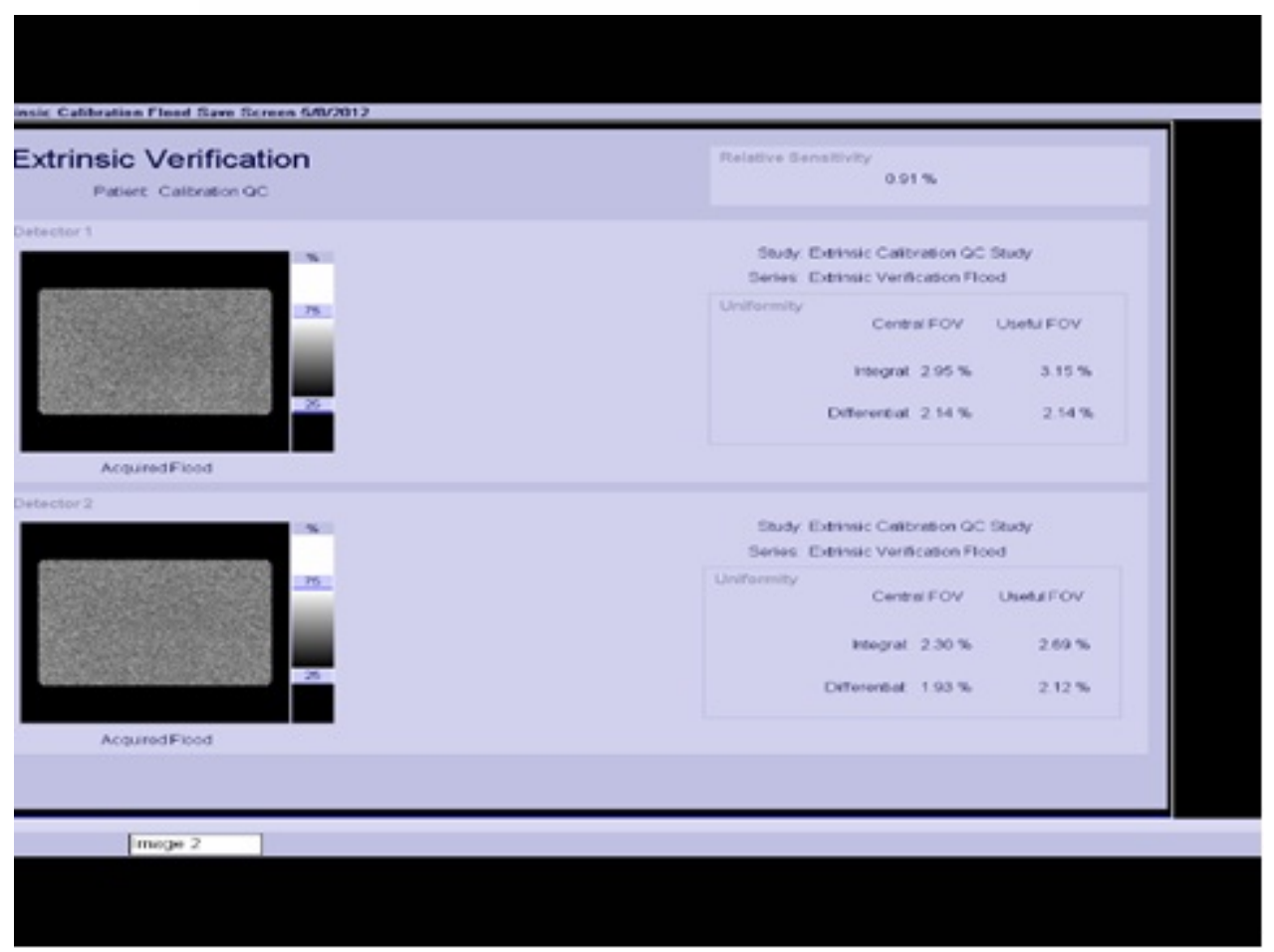

Figure 1: Intrinsic flood calculation image 


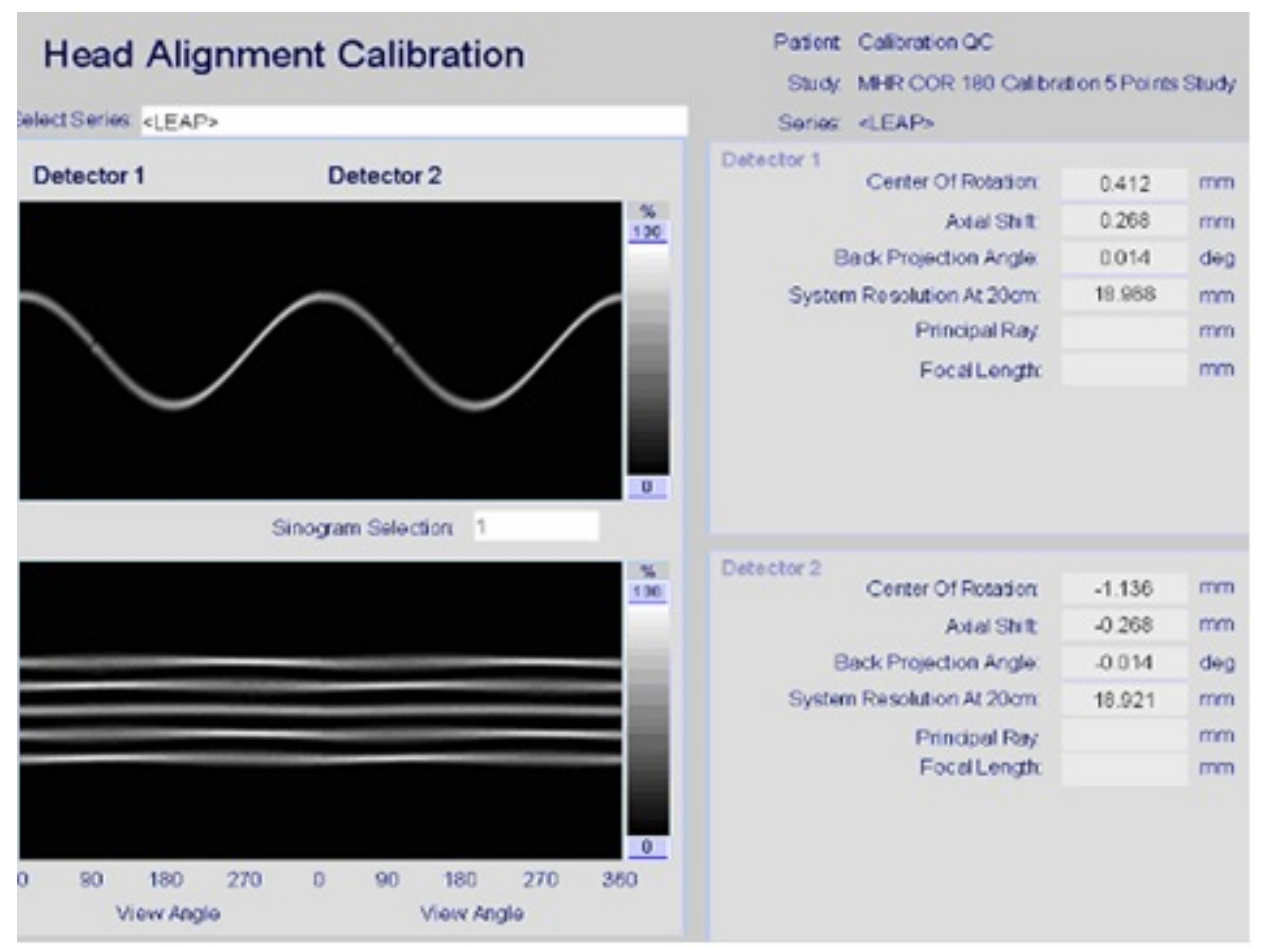

Figure 3: $\mathrm{MHR} / \mathrm{COR} 180^{\circ}$ degree calibration

\section{DISCUSSION}

Intrinsic uniformity includes tuning, peaking, correction and verification of SPECT gamma camera. Manufacturer recommends that these tests should be done on monthly basis. On the other hand, for extrinsic calibration there are no hard and first guidelines unless some damage or replacement of collimator occurs. Extrinsic flood calibration should be performed no more than 10 days following the monthly intrinsic flood calibration or when the machine is re-installed. Measurement of differential uniformity is the most useful indication of the suitability of the SPECT system. Before uniformity correction, modern gamma camera systems typically have integral and differential uniformities between 4-7\% (7). Non-uniformities of this magnitude is generated due to ring artifacts in tomographic data (8), hence all tomographic systems need correction to the raw image data, called "uniformity" correction, before data reconstruction (7).

After uniformity correction, a tomographic system in good working order should have values of differential uniformity in the range $1.0-2.5 \%$ and the integral uniformity a little higher at 1.5- 
$3.5 \%$ (7). Our obtained values correlate well with these ranges. At least, 10kcts/pixel in the flood image needed for uniformity correction (9). In this study, 200 million counts for intrinsic $(1024 \times 1024$ matrix size) and 120 million counts for extrinsic( $1024 \times 1024$ matrix size $)$ uniformity were taken. COR is a very stable parameter in modern gamma camera systems. For high quality tomography, accurate COR correction is important. Errors in COR of as little as 0.5 pixel (2mm) in a 128 x 128 matrix size can result in poor quality image (7). In all configuration angles, our COR values were less than $2 \mathrm{~mm}$ (Table 2). Manufacturer recommends that if the recent calibration deviates by less than 0.5 pixels in $\mathrm{X}$-shift/centre of rotation and Y-shift/axial shift, and less than $0.1^{\circ}$ in the back projection angle, then the calibration of the remaining collimators and configuration may be skipped.

\section{CONCLUSION}

During installation, every gamma camera system needs some recommended QC as acceptance test to build up a baseline values for future quality assurance. The QC values were found in conformity with the manufacturer's specification and the produced images which were used for diagnostic purposes are upto satisfactory level. For long-term performance, manufacturer specified QC should be done in scheduled time.

\section{REFERENCES}

1. James A. Patton and Timothy G. Turkington. SPECT/CT Physical Principles and Attenuation Correction. J NuclMed Technol2008; 36:1-10.

2. S.M.S. Zobly, A.E.O. Osman. Parameters Affecting on Intrinsic Uniformity Test forMEDISO. The Internet Journal of Nuclear Medicine2010; 5(2):DOI: 10.5580/487.

3. American Association of Physicists in Medicine.AAPM Task Group of Nuclear Medicine Committee. Computer-aided scintillation camera acceptance testing 1982.

4. Rizk Abdel-MoneimRizk, Mohamed Anwar Abdelhalim and Hamed I. Farag and Sameh MustafaReda. Effect of widthof energy window on planer and SPECT image uniformity. Retrieved from Medical Physics Website: http://www.docstoc.com/docs/68899073/Effect-of-Window-Width-of-Enter-93k-

5. Mohamed AbdelsattarBayoum. Investigation of Multiple Head Registration / Center of Rotation for SPECT Gamma Cameras. Egyptian Journal of Nuclear Medicine 2009; 2(2): 82-9. 
6. Siemens Operating Instructions Symbia System T16/T6/T2/T Series, Version VA60A.Quality Control and Assurance 2010: 251-323.

7. Michael K. O'Connor, MN. Quality Control of Scintillation Cameras (Planar and SPECT). Retrieved from Medical Physics Website: http:// www.medphysics.wisc.edu/courses/mp573/NM\%3FPET\%20radlab/OConnor.pdf.

8. Rogers WL, Clinthorne NH, Harkness BA. Field flood requirements foremission computed tomography with an Anger camera. J Nucl Med 1982; 23:162-168.

9. Mark W. Groch, William D. Erwin. Sinlge - Photon Emmission Computed Tomography in the year 2011: Instrumentation and Quality Control. J Nucl Med Technol 2011; 29: 915. 